\title{
Multiple Hybrid of Lambda Iteration and Bee Colony Optimization Method for Solving Economic Dispatch Problem
}

\author{
Apinan Aurasopon ${ }^{1}$ and Chiraphon Takeang ${ }^{2}$ \\ ${ }^{1}$ Faculty of Engineering, Mahasarakham University, Mahasarakham Thailand \\ ${ }^{2}$ Dept. of Electrical Engineering, Rajamangala University of Technology Lanna Lampang, \\ Lampang Thailand. \\ apinan.a@msu.ac.th, chiraphon@rmutl.ac.th
}

\begin{abstract}
This article presents a solution to the economic dispatch (ED) problem with a new technique, the multiple of hybrid lambda iteration method and bee colony optimization (MHLBCO) with a smooth cost function. The ED limitations include the electrical energy demand and transmission line losses. The main idea of the MHLBCO algorithm is a method of combining lambda iteration and bee colony optimization then increasing its efficiency by adding multiple search mechanisms. It is an adaptation of the lambda iteration algorithm and bee colony optimization algorithm (HLBCO) to find the best solution in sequence, series, and select the best solution. New techniques have been added to the MHLBCO algorithm to improve the search process, for example the hybrid algorithm, multiple adaptive search scopes and multiple searches. 2 case studies were used to test the efficiency of the MHLBCO algorithm, which included 6 power generating units in case study 1 and 15 power generating units in case study 2 . The optimized results by MHLBCO are compared with those of HLBCO and traditional method approaches, for instance simulated annealing (SA), bee colony optimization (BCO), particle swarm optimization (PSO), krill herd algorithm (KHA), differential evolution algorithm (DE), modified harmony search algorithm (MHSA), hybrid differential evolution algorithm based on particle swarm optimization (DEPSO) and cuckoo search algorithm (CSA). The results of the two case studies confirm that the concepts contained in the MHLBCO algorithm can achieve higher-quality convergence of speed and solutions. The conclusion is this method is used to solve the economic dispatch problem under the objective function, power balance constraint and generator rating constraint efficiently.
\end{abstract}

Keywords: Multiple searches, Lambda iteration, Bee colony optimization, Economic dispatch

\section{Introduction}

Now a day, the power system is constantly evolving. Producing electricity for stability and reliability are important. While the demand for electricity is increasing and the use of electricity at different times has changed cause problems in the planning of electricity. The important thing to consider in generating electricity is the cost of electricity. While each generator generates unequal electricity costs, if the generator works to produce power without a planned power generation, it may incur unnecessary costs. Economics related to electricity generation and transmission can reduce the cost of electricity. It was named Economic Dispatch (ED). The purposes of the problem of ED are to schedule the best results integration of all the units which created to reduce operating costs while addressing load requirements, system parity and inequality. Improving unit output scheduling can reach to significant cost savings.

Aforetime, various methods were developed to solve this problem using mathematical programs and numerical methods for instance lambda iteration method [1], dynamic programming [2], quadratic programming [3], nonlinear programming [4], linear programming [5] and gradient method [6]. It was later found that these methods, when applied to solve ED problems, could not provide optimal solutions. These methods often get stuck in a local optimum. Recently meta-heuristics used to solve problems of ED and power system issues such as Genetic Algorithm (GA) [7]-[9] it mimics the reproduction and survival of organisms

Received: March $25^{\text {th }}, 2019 . \quad$ Accepted: January $19^{\text {th }}, 2021$

DOI: $10.15676 /$ ijeei.2021.13.1.3 
with genetics, Particle Swarm Optimization (PSO) [10]-[12] it mimics the behavior of social and foraging of animals for example birds or fish, Ant Colony Optimization (ACO) [13]-[14] it mimics foraging and the travel of ants to the nearest distance to their destination, Tabu Search (TS) [15] It's a way to find new answers around old answers and prevent them from going back to them. This method remembers the past's answer, forbidding the answer to converge at the same point, Cuckoo Search Algorithm (CSA) [16]-[17] this algorithm is depend on the parasitical cuckoo species behaviour and the flight some birds behaviour, Shuffled Frog Leaping Algorithm (SFLA) [18]-[19] the purpose is to simulate and travesty the behavior of frogs to find food placed on random rocks in the herd, simulation annealing (SA) [20]-[21] to find the answer from the side and move to a new location when the answer value is better than the original and Bee Colony Optimization (BCO) [22]-[24] the BCO algorithms mimic the foraging behavior of bees colony. These methods have acquired a lot of interest from many researchers due to their efficacy to find the best nearly global optimum solution. However, these ones methods also have some downside which limit its performance. The traditional algorithms may have limited access to suitable global optimum solution, when initial solutions are far from suitable solutions may take time to access inappropriate solutions. As a result, with a new population, it can take more time to search for a solution and a long computational time because of the algorithm possesses poor convergence behavior. Therefore, accelerating convergence speed and avoiding the local optimal have become two important. Among the aforementioned methods, there is an efficient way to estimate the global best value of a given function, the BCO method. It has a simple structure which is efficient and employs an advanced search technique. Nevertheless, a traditional BCO algorithm might have a problem of random the initial populations. As a result, it takes more time to search for a solution and takes a long time computational caused the convergence approach is slow. This problem was solved by HLBCO [25], which modified the initial population of the BCO. However, this method might have a problem of narrow in restricting inappropriate search scope. It's difficult to determine the optimum search scopes, so retesting is wasting time. The "multiple search" method was used to solve this problem, with multiple searches helping to find the regions where the most suitable global solution exists. So, the solution in this article is divided into two issues, including initial value estimation and search scope definition. The first one, in the paper,

is to use the total cost of production equality principle $(\lambda)$ as the starting point of the solution,

estimate the initial populations to limited the search scope to find the solution and use BCO to find the most suitable solution around the estimates, that is "hybrid lamda iterations and bee colony optimization (HLBCO)" and second, search optimization to address scoping problems with multiple searches method. This concept is used to improve the execution of traditional $\mathrm{BCO}$ algorithms. This article proposes the new algorithm "multiple hybrid lamda iterations and bee colony optimization (MHLBCO)" to solve the problems of the traditional BCO. MHLBCO introduces additional mechanisms for improving the search process. The possibility study of the MHLBCO demonstrate for solving the static ED problem with a smooth cost function and the best results from the MHLBCO method are compared with conventional methods such as PSO, SA, CSA, the Differential Evolution Algorithm (DE), Modified Harmony Search Algorithm (MHSA), Hybrid Differential Evolution algorithm based on Particle Swarm Optimization (DEPSO) and Krill Herd Algorithm (KHA) in terms of the most appropriate answers.

\section{Economic Dispatch Problem Formulation}

The economic dispatch is identified for four important classifications. These covers are, optimal load flow, ED is an automatic control of power generation, dynamic ED and ED with non-traditional generation sources. Before 1930, There are several methods are used for example, the base unit method, where starts with the most effective production unit to the full capacity, then the second most effective unit starts sorting down, and the best point loading 
method is the start from the highest efficiency unit since it is the unit with the lowest heat rate respectively up to the least efficient unit, that is the highest heat rate unit. In 1930, the incremental cost method later known as the cost equal principle was recognized as producing cost savings. In 1954, computers were used to calculate the loss factor in transmission lines, electronic differential analyzer devices were used in economic scheduling, both on-line and off-line, computers were used to It is planned for production and has been continuously developed until today [26].

Finding the right sum of electricity generation is the primary objective of the ED problem. This is an allocation of load demand commitment for the system to generate a set of generators over a specified period of time with minimal production costs while requiring physical constraints and operational requirements and in accordance with applicable conditions of the system as follows.

\section{A. Objective function}

The primary objective of the ED problem is to reduce the total fuel cost under the electrical system constraints with an objective function that can be expressed as equation (1).

$$
\text { Minimize }: F_{T}=\sum_{i=1}^{N} F_{i}\left(P_{i}\right)
$$

Normally, a thermal unit have had the fuel cost is expressed as a single quadratic function of its output $\left(P_{i}\right)$ as follow (2). It is an equation that represents the smooth cost function, which looks like Figure 1.

$$
F_{i}\left(P_{i}\right)=a_{i} P_{i}^{2}+b_{i} P_{i}+c_{i}
$$

Where $a_{i}, b_{i}$ and $c_{i}$ are the cost coefficients and $P_{i}$ is the power output of the $i^{\text {th }}$ thermal unit.

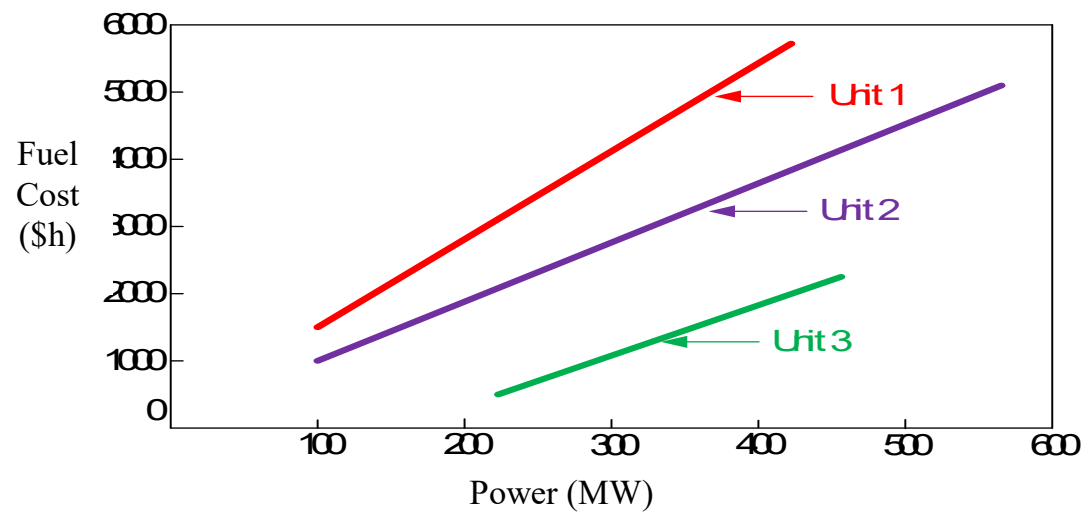

Figure 1. Characteristic of the smooth cost function.

\section{B. Constraints}

The objective function has the following conditions.

a) Constraint of power balance

The sum of the total power produced by all generators must be equal to the sum of the total energy demand and the total power losses on the transmission and distribution lines as follows (3). A

$$
\sum_{i=1}^{N}\left(P_{i}\right)=P_{D}+P_{\text {loss }}
$$

Where $P_{\text {loss }}$ is the total transmission and distribution line losses and $P_{D}$ is the load demand. Ordinarily, in the power system has many factories are located in different locations. All power 
plants are connected by a long transmission line as a network. Power demand is transmitted through the transmission line, causing power loss in the transmission line. Transmission loss is determined by transmission line parameters, bus voltage and load flow. Determining the power loss in a transmission line requires complex calculations. However, an estimate of the power loss in a transmission and distribution line can be reasonably made for a power plant with $N$, the amount of power loss in a transmission and distribution line can be expressed as follows (4).

$$
P_{\text {loss }}=\sum_{i=1}^{N} \sum_{j=1}^{N} P_{i} B_{i j} P_{j}+\sum_{j=1}^{N} B_{0 i} P_{i}+B_{00}
$$

The coefficient matrix $[B]$ is called as loss coefficient matrix or $B$ - coefficient matrix. This matrix is symmetric.

\section{b) Constraint of generator rating}

Each generator shall produce a combined power between its lower and upper boundary conditions, defined by the inequality as follows: (5).

$$
P_{i}^{\min } \leq P_{i} \leq P_{i}^{\max }
$$

Where, $P_{i}^{\max }$ and $P_{i}^{\min }$ are the upper and lower capacity boundary for the $i^{\text {th }}$ generating unit power output, respectively.

\section{Hybrid of Lambda Iteration $(\lambda)$ and Bee Colony Optimization (HLBCO)}

HLBCO is the use of the same principle of equal costs (Increased cost: $\lambda$ ) by setting $\lambda$ as initial, setting new boundaries around $\lambda$ and performing each step by BCO method. At this point, will be describes the BCO, Lambda Iteration $(\lambda)$ and HLBCO for ED problem.

\section{A. Bee colony optimization}

Bee Colony Optimization (BCO) is a way to find the most appropriate value that mimics the foraging of honey bee behaviors. This method divides the bees into two categories, the first group being the scout bees, and the second group is the employee bees to find the answer. Supposing the answer is to find the honey bee source. The function of scout bees is to find random honey bee sources in the scope of possible answers (search space). After the scout bees find the answer, they will fly back to the honey comb to communicate with other bees within the beehive. Bee's communication will use a variety of dances to indicate the amount and direction of the honey. Then, the employee bees will move the honey from the honey source. The numbers of bees will variation to the number of honey bees and the distance.

Before discussing the next episode of how was the $\mathrm{BCO}$, the following of parameter have used the description of the parameters of the $\mathrm{BCO}$ algorithm for general optimization problems.

$n \quad$ is the amount of scout bees.

$m \quad$ is the amount of random honeybee sources from scout bees.

$e \quad$ is the amount of nectar honeybee sources with the highest amount of honeybee selected from $m$

$n_{e p} \quad$ is the amount of employee bees assigned to $e$ honeybee sources.

$n_{s p} \quad$ is the amount of employee bees assigned to $m-e$ honeybee sources. 
The BCO algorithm has a workflow that can be described as follows:

Step 1: $\quad$ BCO algorithm was started with the scout bees $(n)$ being placed randomly in the search space and it subject to the regulatory requirements of the system.

Step 2: The fitness of the sites visited are evaluated by the scout bees and sort the solution from the most to the least.

Step 3: Choose the solution that can be used for a number of $m$ responses from $n$ to find a neighbor solution by determining the bee colony to choose the solution that is the number of $m$.

Step 4: Choose the best solution for the amount of $e$ from $m$ solution. It is a share value of the solution that was selected into two groups. The best solution has the same number of $e$ and followed good solution with the amount of $m-e$.

Step 5: At this stage would be set for employee bees to find a solution in the area of two groups that were divided in step 4 . The $n_{e p}$ employee bees go to find solution surrounding e and the number of $n_{s p}$ employee bees go out for a solution in the area $m-e$.

Step 6: Estimate the value of the answer and compare each other and choose the best approach or solution that matches the given conditions.

Step7: check the threshold for downtime. If conditions are set to be met, then show the most appropriate solution; otherwise, back to step 2.

\section{B. Lambda iteration $(\lambda)$}

This method requires a specific mapping from incremental cost value to each generator's MW output. For alternatives to conventional marginal costs, the generator produces an overall MW of output power, then the method starts with the values of lambda below and above the optimal value, corresponding to all the results that are too small and too much, and then brackets the appropriate value iteratively. To solve the problem, all generators have the same marginal cost, this incremental cost commonly called lambda, the symbol is $\lambda$. When the costs between the generators different, the Lambda method will reduce the capacity, increase the cost and increase the capacity to reduce the cost difference, we can reduce collective costs. If the demand for electric power is changed, the total cost change can be estimated from $\lambda$, use the solved value of $\lambda$ to evaluate $P_{i}$. Equation (2) is the cost of fuel used in the production of each generator power generation. The incremental cost; $(\lambda)$ uses this equation to calculate by using the differential equation, expressed in the Equation (6).

$$
\frac{d\left(F_{i}\left(P_{i}\right)\right)}{d\left(P_{i}\right)}=\lambda
$$

In the ED problem, the fuel cost was the lowest when $\lambda$ was the same. The $\lambda$ value is calculated for the starting point of the system by equation (7) and calculate the electric power of each generator from equation (8).

$$
\begin{gathered}
\lambda=\frac{P_{D}+\sum_{i=1}^{N} \frac{b_{i}}{2 a_{i}}}{\sum_{i=1}^{N} \frac{1}{2 a_{i}}} \\
P_{i}=\frac{\lambda-b_{i}}{2 a_{i}}
\end{gathered}
$$




\section{Hybrid of lambda iteration ( $\lambda$ ) and bee colony optimization (HLBCO) for ED problem [25]}

The BCO method can obtain a global optimum within a short time and guarantee an optimum solution. However, in these techniques the initial populations are generated randomly. This results in long computation times and a long time to convergence when the generated initial populations are too far from the optimum solution. This problem has been solved by HLBCO in which the initial BCO population was modified. The HLBCO uses the equal cost principle or incremental cost: $\lambda$ to solve the ED problem, estimate the initial value and cramped the search, then use the good properties of the BCO method to search for answers around the set limits. The HLBCO algorithm for solving ED problems can be described in steps hereinafter:

Step 1: Determine the parameters for the HLBCO algorithm.

Step 2: Using equation (7), calculate the $\lambda$ value and use it as the initial value.

Step 3: Using equations (9) and (10) calculate the minimum and maximum search boundary values of each generator.

$$
\begin{aligned}
& P_{i}^{\min }=\frac{\lambda-b_{i}}{2 a_{i}}-\left(\frac{\lambda-b_{i}}{2 a_{i}} \times \text { rank }\right) \\
& P_{i}^{\max }=\frac{\lambda-b_{i}}{2 a_{i}}+\left(\frac{\lambda-b_{i}}{2 a_{i}} \times \text { rank }\right)
\end{aligned}
$$

rank is between 0 and 1 and is a factor to determine the size of the search scope.

Step 4: Randomizes the initial population value $(n)$, provided within the specified conditions and scope.

Step 5: Evaluate the initial population fitness value and arrange it in ascending order.

Step 6: Select the best $(m)$ solutions by sorting and dividing the solution into two groups: $(e)$ are the best solution and $(m-e)$ are the next best solution.

Step 7: Set the search scope around each solution grouped in step 6.

Step 8: Send $n_{e p}$ bees swarms to find solutions in group e and $n_{s p}$ bees swarms for solution in group $(m-e)$

Step 9: Create new solutions in the $e$ and $m-e$ solutions group, where the amount of solutions in group $e$ is equal to $n_{e p}$, the amount of solutions in the $m$-e group is equal to $n_{s p}$, evaluate their suitability and select the optimum solution.

Step 10: Check the suitability of the answer, if within the required criteria, stop and if not, go back to step 4 .

Step 11: Use the most suitable solution as the output power of each generator.

\section{Multiple Hybrid of Lambda Iterations and Bee Colony Optimization (MHLBCO) for ED Problem}

Multiple searches provide promising powerful searches leading to the most affordable global solution. MHLBCO use a sorted search method based on a given number of algorithms in a single run. The main idea of MHLBCO arose from the HLBCO solution in section 3.3. It is difficult to determine the rank configuration in the 3rd step to obtain the optimum value, requiring multiple tests which are time consuming. Convergent speed can be improving by using multiple searches. The MHLBCO algorithm solves the problem of convergence speed to increase accessibility with the global solution. Figure 2 shows several independent HLBCO algorithms including independently adjustable rank, which work together as an MHLBCO algorithm with additional steps and mechanisms are as follows. 


\section{A. Specify MHLBCO parameter}

Determining the parameter of a bee colony is to determine the number of scout bees the amount of $\mathrm{n}$ that will be released to find the honey bee sources. It is to determine the amount of output power that is subject to the constraints of each generator, which is equivalent to a number of scout bees emitted. In this paper, the parameter value of how was BCO, HLBCO and MHLBCO are used to test the operation is presented in table 1.

Table 1. The parameters of HLIBCO

\begin{tabular}{lc}
\hline \hline Parameters & Number \\
\hline Initial populations sites $(n)$ & 20 \\
Selected sites $(m)$ & 10 \\
Selected best sites $(e)$ & 5 \\
Bees all around other sites $\left(n_{s p}\right)$ & 50 \\
Bees all around best sites $\left(n_{e p}\right)$ & 50 \\
\hline \hline
\end{tabular}

\section{B. The Lambda Iteration Value ( $\lambda$ ) Calculation}

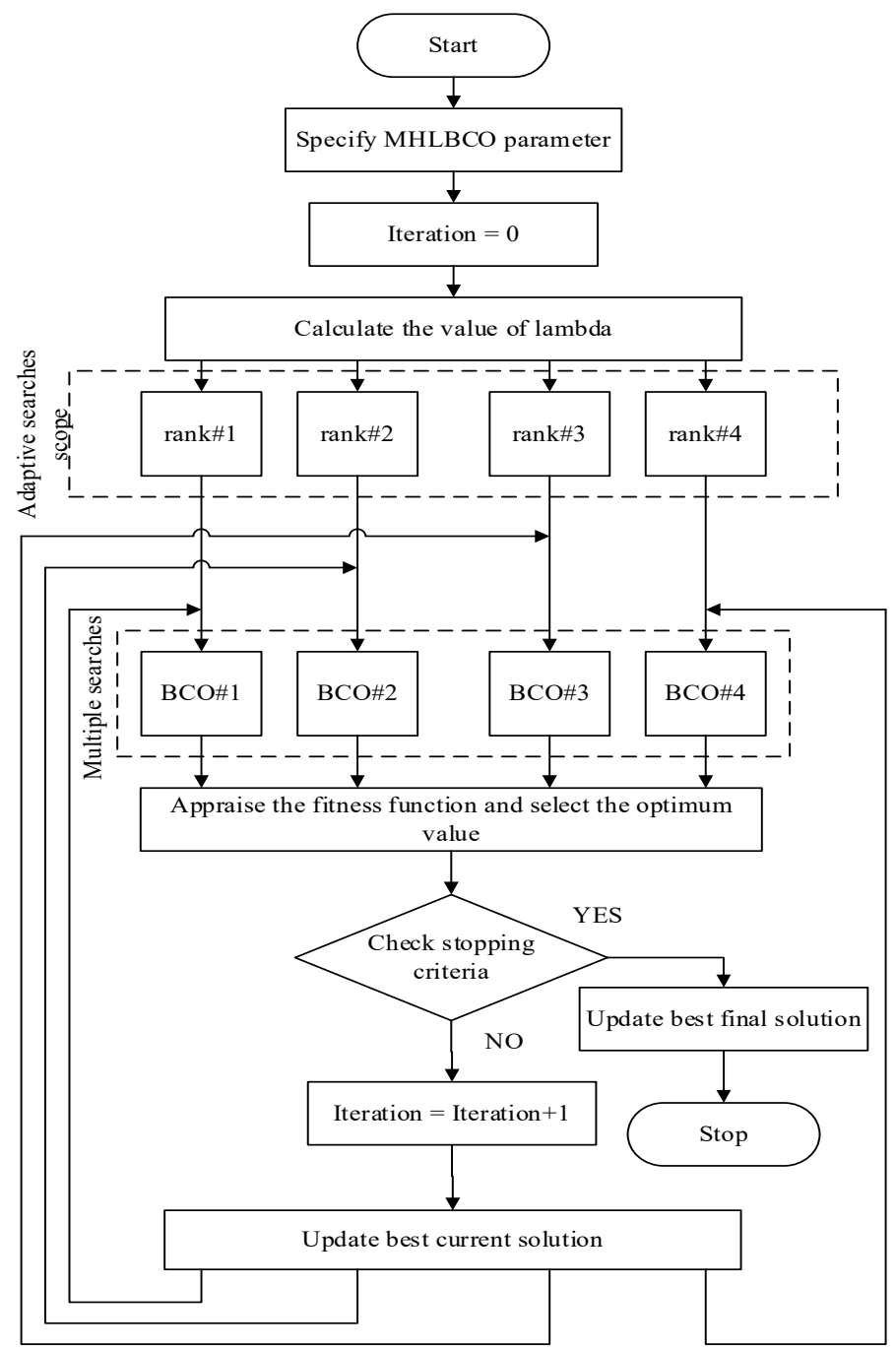

Figure 2. Procedure of MHLBCO 
$\lambda$ is incremental cost principle using the equal cost principle, estimated the starting value to cramped the search area and to use the good features of the BCO approach to find answers within new areas. The total cost of production is lowest when $\lambda$ is the same. The value of $\lambda$ can be calculated from the equation where (7).

\section{Multiple adaptive search scope}

In this article, the scope of finding the answer has been redefined with rank which is the factor used to define the upper and lower search boundaries of the $i^{\text {th }}$ generating unit that calculated from equations (9) and (10), using $\lambda$ as the initial value. The result of finding the answer depends on the magnitude of rank. It is difficult to determine the proper rank sizing, it is necessary to perform multiple tests which will take a long time. Multiple rank scaling at the same time is a multi-adaptive search scope. The search process within a multi-adaptive search scope increases the chances of finding the global optimum solution and quickly converging to the right one. The size of the rank is important in these processes. The key factor is the range of variance within the scope of the search, which depends on the magnitude of rank. Accordingly, rank must be properly defined. Conventionally, the rank size with low value, the search scope is narrow and time find the right answer will be less. On the contrary, a wider rank size is a more accurate solution but results in longer computation times. However, the optimum answer may be the local optimum. This article presents, rank size can be adjusted according to the amount of bee colony, which makes it more accurate to fix the problem. Therefore, multi-adaptive search scope improves the answer-finding process, increases computational efficiency and speed up convergence to the right solution. In the presented algorithm, configure the rank values as follows: $\operatorname{rank} \# 1=0.05$, $\operatorname{rank} \# 2=0.1$, $\operatorname{rank} \# 3=0.15$ and $\operatorname{rank} \# 4=0.2$.

\section{Multiple search}

Large solutions can be achieved by using multiple computers to perform calculations at the same time known as parallel search. Howsoever, the sequential search used in this document is performed on a single computer [27]. Reducing the time, it takes to find the right solution by improving the search process and the scope of each adjustable search as well as the capability to manipulate results are some of the basic concepts in this article. Multiple searches are a sorted search method that combines additional features with search algorithms and capabilities. Multiple searches allow multiple answers at the same time. The best answers are selected from a single algorithm. Multiple searches are the way to take advantage of the properties of multiple algorithms that are still incomplete in traditional algorithms and help find an answer that is likely to be the global optimum solution exists. This idea made the BCO algorithm get a more optimized mechanism after taking the obtained values from the lambda iterations and defining the span of multiple search scopes as described in section 4.3. Important mechanisms are implemented in the BCO algorithm for better performance as described in section 3.2. The algorithm work is sorted from $\mathrm{BCO} \# 1$ to $\mathrm{BCO} \# 4$, then compares the answers obtained from all algorithms and selects the best answer.

\section{E. Appraise the fitness and choose the optimum solution}

The idea at this stage is to remove the worst design solution after the end of each simulation cycle and select the best solution from the best design solution. Good design requires a good fitness function to indicate proximity to overall requirements and use fitness function to test or simulate the results obtained from the solution. Poor design will cause the algorithm to find an unusual solution or there will be convergent problems. Moreover, it must also be computed quickly. All are processes of evaluating the performance and selecting of the most appropriate answer. 


\section{F. Check the stopping criteria}

Typically, there are several possible downtime conditions. In the algorithm presented here, there is a search stop condition if three conditions are met. Firstly, when the algorithm has run until the specified time that is if the search process is ongoing until it takes more than or equal to the specified time, it will stop. Second, it depends on the predefined number of iteration cycles, if the iteration is equal to or greater than the specified number of iterations, the search process stops. Finally, when the appropriate value meets the objective function and under the system's limitations and conditions, the answer search stops.

\section{Case Study}

To evaluate the operation of the MHLBCO algorithm, it was applied to economic dispatch problems in two case studies with large and medium-sized systems. The test case studies consisted of a system with the 6 unit generators and a system with the 15 unit generators. The algorithm performance testing was implemented in a MATLAB program running on TOSHIBA Satellite P745, Intel Core i5, $2.30 \mathrm{GHz}$ with 8 GB RAM.

\section{A. The first case study}

The system used to test for the first case consisted of six thermal units as well as a generating limit, a power balance limit, a generator rating limit, 46 transmission lines and 26 buses. It needs the electric power of $1263 \mathrm{MW}$. The generator feature of each is shown in table 2 and the B-coefficient matrix as follow [27].

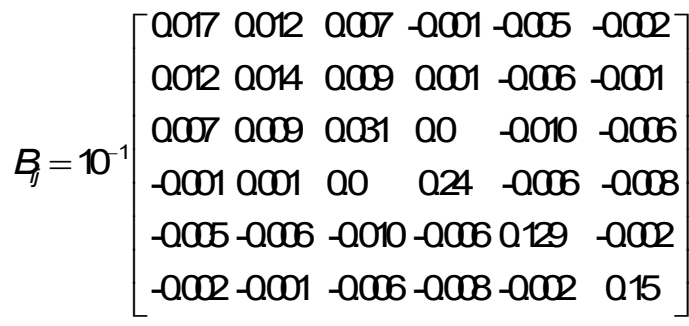

$$
\begin{aligned}
& B_{i}=10^{-3} \times[-03908-01297070470059102161-06635] \\
& B_{0}=[0056]
\end{aligned}
$$

Table 2. Generator data in case 1

\begin{tabular}{lcllll}
\hline \hline Unit & $a_{i}$ & $b_{i}$ & $c_{i}$ & $P_{i}^{\min }$ & $P_{i}^{\max }$ \\
\hline 1 & 0.0070 & 7.00 & 240 & 100 & 500 \\
2 & 0.0095 & 10.0 & 200 & 50 & 200 \\
3 & 0.0090 & 8.50 & 220 & 80 & 300 \\
4 & 0.0090 & 11.0 & 200 & 50 & 150 \\
5 & 0.0080 & 10.5 & 220 & 50 & 200 \\
6 & 0.0075 & 12.0 & 190 & 50 & 120 \\
\hline \hline
\end{tabular}

\section{B. The second case study}

This system consists of 15 generators, with the characteristics shown in Table 3. All generators have a power requirement of $2630 \mathrm{MW}$, including the transmission loss of the B-coefficient matrix as shown in the references [28]. 
Table 3. Generator data in case 2

\begin{tabular}{cccccc}
\hline \hline Unit & $P_{i}^{\min }$ & $P_{i}^{\max }$ & $a_{i}$ & $b_{i}$ & $c_{i}$ \\
\hline 1 & 150 & 455 & 0.000299 & 10.1 & 671 \\
2 & 150 & 455 & 0.000183 & 10.2 & 574 \\
3 & 20 & 130 & 0.001126 & 8.80 & 374 \\
4 & 20 & 130 & 0.001126 & 8.80 & 374 \\
5 & 150 & 470 & 0.000205 & 10.4 & 461 \\
6 & 135 & 460 & 0.000301 & 10.1 & 630 \\
7 & 135 & 465 & 0.000364 & 9.80 & 548 \\
8 & 60 & 300 & 0.000338 & 11.2 & 227 \\
9 & 25 & 162 & 0.000807 & 11.2 & 173 \\
10 & 25 & 160 & 0.001203 & 10.7 & 175 \\
11 & 20 & 80 & 0.003586 & 10.2 & 186 \\
12 & 20 & 80 & 0.005513 & 9.90 & 230 \\
13 & 25 & 85 & 0.000371 & 13.1 & 225 \\
14 & 15 & 55 & 0.001929 & 12.1 & 309 \\
15 & 15 & 55 & 0.004447 & 12.4 & 323 \\
\hline \hline
\end{tabular}

\section{Simulation Results}

MHLBCO and HLBCO were tested to assess performance with two ED problematic power systems with 6 and 15 generators. All optimization methods (BCO, PSO, and SA) were used in comparison with different solutions obtained from random. To assess the effectiveness of each method, all search algorithms are executed at the same time interval. So, the fastest convergence would be a powerful way. To compare the effectiveness of all methods, convergence speed, elapsed time, and the results of the answers were used to evaluate performance. In this performance evaluation, the parameters of BCO, HLBCO and MHLBCO are shown in Table 1 and the parameters are used in the PSO and SA methods shown in Table 4 and Table 5. The configurations in Table 1, Table 4-5 were generated on several times iterative trials, taking into account the speed of finding answers and the quality of the answers.

Table 4. The parameters of PSO

\begin{tabular}{lc}
\hline \hline Parameters & Number \\
\hline Population size $(N)$ & 300 \\
Generations & 100 \\
Acceleration constant $\left(C_{1}\right),\left(C_{2}\right)$ & $1.9,1.9$ \\
Inertia weight factor $\left(W_{\max }\right),\left(W_{\min }\right)$ & $0.9,0.4$ \\
Limit of change in velocity $\left(V_{\max }\right),\left(V_{\min }\right)$ & $0.5 P^{\max },-0.5 P^{\min }$ \\
\hline \hline
\end{tabular}

Table 5. The parameters of SA

\begin{tabular}{lc}
\hline \hline Parameters & Number \\
\hline Cool Shed & 0.83 \\
incipient temperature & 500 \\
Last temperature & 1 \\
Maximal number of successes within & 50 \\
Maximal number of tries & 1000 \\
\hline \hline
\end{tabular}

\section{A. Simulation results in case 1}

Five methods (MHLBCO, HLBCO, BCO, PSO, and SA), two case studies were scheduled to be performed on the entire system. In this test system, $P g$ is the sum of the output power of 6 
generators: $P_{1}, P_{2}, P_{3}, P_{4}, P_{5}$ and $P_{6}$. For the 1,263 MW electricity demand, the results of the MHLBCO and HLBCO methods were compared with the obtained results with the BCO, PSO and SA algorithms. In terms of minimum generation cost and computation efficiency, the methods that offer their best solution are shown in Table 6 . The convergent speeds of the MHLBCO and HLBCO methods compared with other methods for 6 units system are shown in Figure 3. Clearly, the minimum generation cost of the MHLBCO and HLBCO methods have a similar value and lowest in comparison to other methods and the convergence speeds of the MHLBCO to the optimum solution is faster than other methods. The results of the MHLBCO method are compared with the CSA [29], MHSA [30] and DE [31] methods (Table 7).

Table 6. Results of six units system in case 1

\begin{tabular}{cccccc}
\hline \hline Unit output & MHLBCO & HLBCO & BCO & PSO & SA \\
\hline$P_{1}(\mathrm{MW})$ & 451.34 & 451.53 & 415.01 & 452.61 & 454.17 \\
$P_{2}(\mathrm{MW})$ & 173.76 & 173.14 & 172.71 & 171.90 & 168.55 \\
$P_{3}(\mathrm{MW})$ & 257.61 & 260.19 & 261.90 & 259.21 & 257.65 \\
$P_{4}(\mathrm{MW})$ & 137.45 & 136.07 & 138.33 & 138.56 & 133.94 \\
$P_{5}(\mathrm{MW})$ & 163.72 & 163.77 & 165.21 & 166.37 & 162.70 \\
$P_{6}(\mathrm{MW})$ & 91.24 & 90.47 & 86.04 & 85.59 & 98.14 \\
PT $(\mathrm{MW})$ & 1275.12 & 1275.17 & 1275.21 & 1275.24 & 1275.14 \\
FT $(\$ / \mathrm{h})$ & 15439.50 & 15439.56 & 15439.87 & 15440.53 & 15440.60 \\
PLosses & 12.12 & 12.17 & 12.20 & 12.24 & 12.14 \\
Time $(\mathrm{s})$ & 18.78 & 4.77 & 7.35 & 14.45 & 10.24 \\
\hline \hline
\end{tabular}

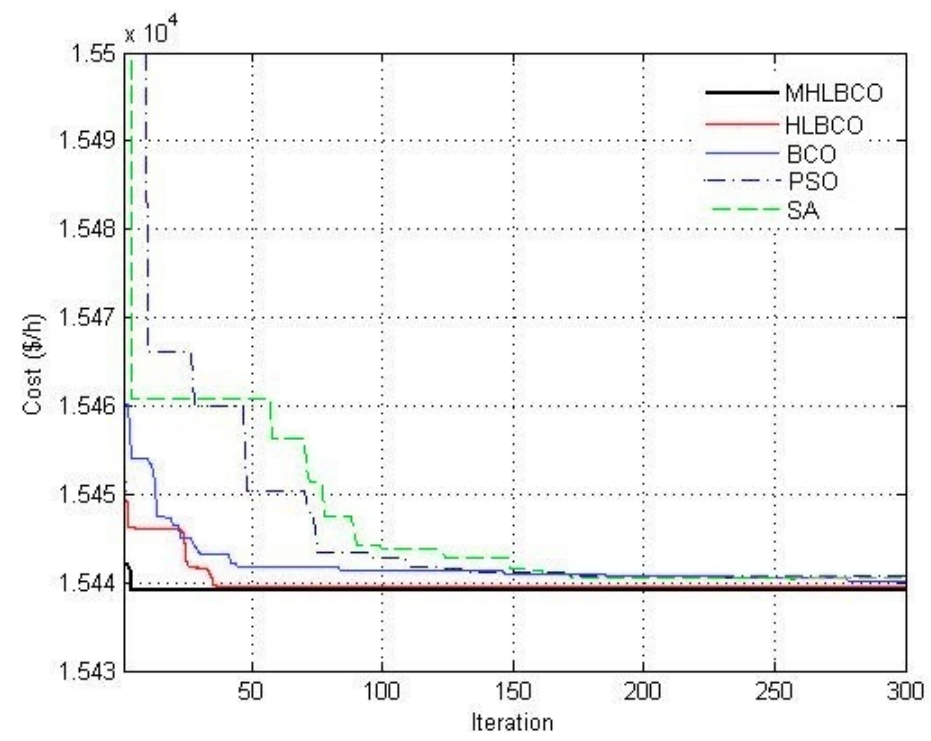

Figure 3. Convergence curve of 6 unit system.

Table 6 shows that the MHLBCO and HLBCO algorithms performed better in finding the most suitable answer in the search area compared to BCO, PSO and also the proposed SA method. The best solutions are presented in this table. The optimum cost obtained by the MHLBCO is 15439.50 , which compares favorably with the other results in the table. By comparison, in terms of time, this method was presented at the longest in 18.78 seconds which was achieved by using the HLBCO method, arranged in series to find the answers and selecting the best solution. However, Figure 3 shows the convergent speeds of the MHLBCO, HLBCO, $\mathrm{BCO}, \mathrm{PSO}$ and SA. The SA converged to the optimum cost from 150 iterations onwards, PSO 
converged to the optimum cost from 180 iterations onwards, $\mathrm{BCO}$ converged to the optimum cost from 270 iterations onwards, HLBCO converged to the optimum cost from 30 iterations onwards, whereas MHLBCO converges at less than 10 iterations. It can see that the level of the cost function doesn't change too much while MHLBCO has the fastest convergence speed. Similarly, the cost function that achieved by the MHLBCO method was significantly better than those obtained by the CSA, MHSA, and DE methods (Table 7).

Table 7. Results and comparison to other optimization methods evaluated in case 1

\begin{tabular}{ccccc}
\hline \hline Unit output & MHLBCO & CSA[29] & MHSA[30] & DE[31] \\
\hline$P_{1}(\mathrm{MW})$ & 451.34 & 447.48 & 446.73 & 448.27 \\
$P_{2}(\mathrm{MW})$ & 173.76 & 173.22 & 173.49 & 172.96 \\
$P_{3}(\mathrm{MW})$ & 257.61 & 263.38 & 263.76 & 263.44 \\
$P_{4}(\mathrm{MW})$ & 137.45 & 138.95 & 138.83 & 139.30 \\
$P_{5}(\mathrm{MW})$ & 163.72 & 165.41 & 165.65 & 165.28 \\
$P_{6}(\mathrm{MW})$ & 91.24 & 87.00 & 86.95 & 86.68 \\
PT $(\mathrm{MW})$ & 1275.12 & 1275.45 & 1275.42 & 1275.93 \\
FT $(\$ / \mathrm{h})$ & 15439.50 & 15443.08 & 15442.52 & 15449.58 \\
PLosses $^{-}$ & 12.12 & 12.45 & 12.42 & 12.95 \\
\hline \hline
\end{tabular}

B. Simulation results in case 2

This system consists of 15 thermal power generators. All thermal power generators must support $2630 \mathrm{MW}$ of load requirements. The results of the MHLBCO and HLBCO methods are compared the answer with the obtained results from BCO, PSO, and SA in terms of minimum generation cost and computation efficiency. This sample has a rather problematic search area compared to the previous example. After the proposed algorithm performance test, the results of the test are shown in Table 8, according to system conditions and regulations. The results of the MHLBCO algorithm are compared with the DEPSO [32] and KHA [33] algorithm (Table 9).

Table 8. Results of fifteen units system in case 2

\begin{tabular}{clllll}
\hline \hline Unit output & MHLBCO & HLBCO & BCO & PSO & SA \\
\hline$P_{1}(\mathrm{MW})$ & 438.52 & 450.30 & 424.46 & 452.36 & 416.19 \\
$P_{2}(\mathrm{MW})$ & 427.92 & 444.58 & 448.56 & 437.74 & 434.84 \\
$P_{3}(\mathrm{MW})$ & 123.21 & 127.02 & 129.64 & 122.80 & 128.50 \\
$P_{4}(\mathrm{MW})$ & 123.56 & 128.52 & 129.55 & 128.41 & 129.10 \\
$P_{5}(\mathrm{MW})$ & 329.02 & 286.20 & 295.61 & 254.09 & 352.91 \\
$P_{6}(\mathrm{MW})$ & 439.87 & 457.43 & 434.74 & 453.66 & 434.57 \\
$P_{7}(\mathrm{MW})$ & 462.08 & 455.19 & 462.88 & 449.21 & 462.50 \\
$P_{8}(\mathrm{MW})$ & 60.67 & 61.42 & 68.73 & 61.68 & 61.37 \\
$P_{9}(\mathrm{MW})$ & 26.21 & 33.18 & 28.28 & 28.15 & 28.92 \\
$P_{10}(\mathrm{MW})$ & 29.24 & 57.61 & 55.23 & 84.90 & 30.61 \\
$P_{11}(\mathrm{MW})$ & 76.57 & 51.97 & 51.22 & 55.26 & 72.72 \\
$P_{12}(\mathrm{MW})$ & 60.61 & 49.10 & 71.65 & 70.93 & 52.40 \\
$P_{13}(\mathrm{MW})$ & 26.58 & 25.72 & 25.23 & 26.18 & 25.16 \\
$P_{14}(\mathrm{MW})$ & 16.60 & 15.76 & 18.36 & 15.99 & 15.40 \\
$P_{15}(\mathrm{MW})$ & 15.28 & 15.18 & 15.04 & 17.14 & 15.78 \\
PT (MW) & 2655.94 & 2659.18 & 2659.18 & 2658.51 & 2660.94 \\
FT (\$/h) & 32546.05 & 32569.54 & 32572.94 & 32585.37 & 32584.24 \\
PLsses $_{\text {Time }(\mathrm{s})}$ & 35.94 & 29.18 & 29.18 & 28.51 & 30.94 \\
\hline \hline
\end{tabular}


Table 8 shows that the MHLBCO and HLBCO algorithms performed better in finding the most appropriate answer in the search area compared to BCO, PSO and also the proposed SA method. The best answer to each method is presented in this table. The lowest total cost that MHLBCO can give the best results is 32546.05 , which compares favorably with the other results in the table. Figure 4 shows a comparison of the convergent speeds of MHLBCO, HLBCO, BCO, PSO and SA. The SA converged to the optimum cost from 280 iterations onwards, PSO converged to the optimum cost from 240 iterations onwards, BCO converged to the optimum cost from 260 iterations onwards, HLBCO converged to the optimum cost from 140 iterations onwards, whereas MHLBCO converges at less than 6 iterations. It can be seen that the level of the cost function doesn't change too much, while MHLBCO has the fastest convergence speed. Similarly, the cost function that achieved by the MHLBCO method was significantly better than those obtained by the DEPSO and KHA methods (Table 9).

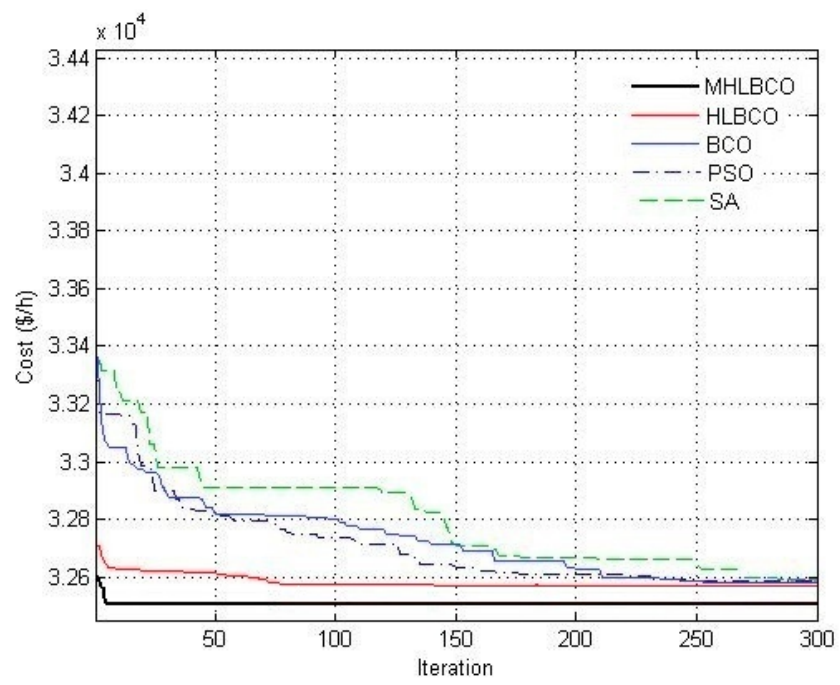

Figure 4. Convergence curve of 15 unit system

Table 9. Results and comparison to other optimization methods evaluated in case 2

\begin{tabular}{cccc}
\hline \hline Unit output & MHLBCO & DEPSO[32] & KHA[33] \\
\hline$P_{1}(\mathrm{MW})$ & 438.52 & 450.30 & 424.46 \\
$P_{2}(\mathrm{MW})$ & 427.92 & 444.58 & 448.56 \\
$P_{3}(\mathrm{MW})$ & 123.21 & 127.02 & 129.64 \\
$P_{4}(\mathrm{MW})$ & 123.56 & 128.52 & 129.55 \\
$P_{5}(\mathrm{MW})$ & 329.02 & 286.20 & 295.61 \\
$P_{6}(\mathrm{MW})$ & 439.87 & 457.43 & 434.74 \\
$P_{7}(\mathrm{MW})$ & 462.08 & 455.19 & 462.88 \\
$P_{8}(\mathrm{MW})$ & 60.67 & 61.42 & 68.73 \\
$P_{9}(\mathrm{MW})$ & 26.21 & 33.18 & 28.28 \\
$P_{10}(\mathrm{MW})$ & 29.24 & 57.61 & 55.23 \\
$P_{11}(\mathrm{MW})$ & 76.57 & 51.97 & 51.22 \\
$P_{12}(\mathrm{MW})$ & 60.61 & 49.10 & 71.65 \\
$P_{13}(\mathrm{MW})$ & 26.58 & 25.72 & 25.23 \\
$P_{14}(\mathrm{MW})$ & 16.60 & 15.76 & 18.36 \\
$P_{15}(\mathrm{MW})$ & 15.28 & 15.18 & 15.04 \\
PT $(\mathrm{MW})$ & 2655.94 & 2659.18 & 2659.18 \\
FT $(\$ / \mathrm{h})$ & 32546.05 & 32569.54 & 32572.94 \\
P & 25.94 & 29.18 & 29.18 \\
\hline \hline
\end{tabular}




\section{Conclusion}

The proposed algorithm introduces a new mechanism for ED problems with different case studies. Many more complex techniques have been added to the proposed algorithms to increase efficiency, such as multiple searches, adaptive searches scope, initial estimation using the lambda iteration and more importantly, this algorithm has been optimized by finding the most suitable solution by means of the BCO into MHLBCO to increase its search potential. Optimizing the MHLBCO through the proposed techniques and mechanisms, MHLBCO outperforms other recently reported algorithms. The efficiency of the algorithm that was presented can be evidenced in both of the ED case studies. It is obvious from the convergent quality of MHLBCO algorithm in two case studies, the robustness of the algorithm is proved. The results of tests and comparisons in a system consisting of 6 and 15 generators confirmed that MHLBCO outperformed the HLBCO, BCO, PSO and SA methods in terms of the lowest production costs, the efficiency of this algorithm was high, while the method offers stable convergence characteristics and good computing performance. By using the MHLBCO method, execution time could also be reduced. In case studies, the proposed method produced better results in comparison with the CSA, MHSA, DE, DEPSO and KHA methods depending on the test conditions that were evaluated. The numbers of the results obtained from the algorithm, which presented clearly show that this algorithm yields better answers. The power system operator can use this algorithm to guide the efficient reduction of the total cost of generator power generation.

\section{Acknowledgements}

This research project is financially supported by Mahasarakham University, Thailand (Fast Track 2020). Thanks Prof. Ian Warrington, for valuable comments and suggestions.

Authors: The authors are with the Electronics for Agriculture Research Unit, Faculty of Engineering, Mahasarakham University, Thailand and Faculty of Engineering, Rajamangala University of Technology Lanna, Lampang, Thailand. The correspondence address is: E-mail: Chiraphon@rmutl.ac.th

\section{References}

[1]. A. J. Wood and B. F. Wollenberg, "Power Generation Operation and Control". New York: Wiley, 1984.

[2]. R.J. Ringlee and D.D. Williams, "Economic dispatch operation considering valve throttling losses II-distribution of system loads by the method of dynamic programming," IEEE Trans. Power Appar. Syst. vol. 81. Issue 3, pp. 615-620, 1963.

[3]. L. d. Santos Coelho and V. C. Mariani: "Combining of Chaotic Differential Evolution and Quadratic Programming for Economic Dispatch Optimization with Valve-Point Effect," IEEE Transactions on Power Systems, vol. 21, no. 2, pp. 989-996, 2006.

[4]. A. M. Sasson, "Nonlinear Programming Solutions for Load-Flow, Minimum-Loss, and Economic Dispatching Problems," IEEE Transactions on Power Apparatus and Systems, vol. pas-88, no. 4, pp. 399-409, 1969.

[5]. A. Farag, S. Al-Baiyat and T. C. Cheng, "Economic Load Dispatch Multi objective Optimization Procedures Using Linear Programming Techniques," IEEE Transactions on Power Systems, vol. 10, no. 2, pp. 731-738, 1995.

[6]. M. Ramamoorty, R. N. Dhar, and P. K. Mukherjee, "Reduced-gradient method for economic dispatch," in Electrical Engineers Proceedings of the Institution of Electrical Engineers, vol. 120, Issue 11, pp.1419-1420, 1973.

[7]. V. Kumar, J. Singh, Y. Singh, and S. Sood, "Optimal Economic Load Dispatch Using Genetic Algorithms," International Journal of Electrical, Computer, Energetic, Electronic and Communication Engineering, vol. 9, no. 4, pp. 463-470, 2015.

[8]. S. A. Oluwadare, G. B. Iwasokun, O. Olabode, O. Olusi, and A. E. Akinwonmi, "Genetic Algorithm-based Cost Optimization Model for Power Economic Dispatch Problem," 
British Journal of Applied Science \& Technology, vol. 15, Issue 6, pp.1-10, 2016.

[9]. A. Q. Al-Shetwi and M. I. Alomoush, "A New Approach to the Solution of Economic Dispatch using Genetic Algorithm," Journal of Engineering and Technology, vol. 7, no. 1, pp. $40-48,2016$.

[10]. K. Srikanth and V. HariVamsi, "Partical Swarm Optimization Technique for Dynamic Economic Dispatch," International Journal of Research in Engineering and Technology, vol. 5, Issue 5, pp. 460-466, 2016.

[11]. H. Zhang, "An Improved Particle Swarm Optimization Algorithm for Dynamic Economic Dispatch Problems," International Journal of Innovative Research in Engineering \& Management (IJIREM), vol. 3, Issue 4, pp.264-266, 2016.

[12]. H. Lotfi, A. Dadpour, and M. Samadi, "Solving Economic Dispatch in Competitive Power Market Using Improved Particle Swarm Optimization Algorithm," International Journal of Smart Electrical Engineering, vol.6, no.1, pp. 35-41, 2017.

[13]. M. N. Nwohu and O. O. Paul, "Evaluation of Economic Load Dispatch Problem in Power Generating Stations by the Use of Ant Colony Search Algorithms," International Journal of Research Studies in Electrical and Electronics Engineering (IJRSEEE), vol. 3, Issue 1, pp. 20-29, 2017.

[14]. S. Vig and B. S. Surjan, "Optimal Power Dispatch of WECS and UPFC with ACO and ANFIS Algorithms," International Journal on Electrical Engineering and Informatics (IJEEI), vol. 10, Issue 1, pp. 14-36, 2018.

[15]. V. K. Joshi, "Optimization of Economic Load Dispatch Problem by using Tabu Search Algorithm," International Journal of Latest Trends in Engineering and Technology, vol. 8, Issue 4-1, pp.182-187, 2017.

[16]. K. Chandrasekaran, S. P. Simon, and N. P. Padhy, "Cuckoo Search Algorithm for Emission Reliable Economic Multi-Objective Dispatch Problem," IETE Journal of Research, vol. 60, Issue 2, pp.128-138, 2014.

[17]. T. T. Nguyen, D. N. Vo, and B. H. Dinh, "Cuckoo Search Algorithm Using Different Distributions for Short-Term Hydrothermal Scheduling with Reservoir Volume Constraint," International Journal on Electrical Engineering and Informatics (IJEEI), vol. 8, pp. 76-92, 2017.

[18]. M. Darabian, S. M. Moheni-Bonab, and B. Moohammadi-Ivatloo, "Improvement of Power System Stability by Optimal SVC Controller Design Using Shuffled Frog Leaping Algorithm," IETE Journal of Research, vol. 61, Issue 2, pp.160-169, 2015.

[19]. K. Selvakumar, K. Vijayakumar, D. Sattianadan, and C. S. Boopathi, "Shuffled Frog Leaping Algorithm (SFLA) for Short Term Optimal Scheduling of Thermal Units with Emission Limitation and Prohibited Operational Zone (POZ) Constraints," Indian Journal of Science and Technology, vol. 9, no. 42, pp. 1-6, 2016.

[20]. M. Venkatesh and R. Raghutu, "Economic Load Dispatch using Simulated Annealing Algorithm," International Research Journal of Engineering and Technology (IRJET), vol. 2, Issue 3, pp. 1961-1964, 2015.

[21]. Z. Ismail, B. Farid, and G. Amel, "Simulated Annealing Optimization for Generation Scheduling with Cubic Fuel Cost Function," WSEAS Transactions on Information Science and Applications, vol. 14, pp. 64-69, 2017.

[22]. E. Assareh and M. Biglari, "A Novel Approach to Capture the Maximum Power Generation from Wind Turbines Using Hybrid MLP Neural Network and Bees Algorithm (HNNBA)," IETE Journal of Research, vol. 62, Issue 3, pp. 368-378, 2015.

[23]. N. S. Tung and S. Chakravorty, "Solution to Economic Power Dispatch Planning Problem considering Generator Constraints using Artificial Bee Colony Algorithm," International Journal of Hybrid Information Technology, vol. 9, no. 5, pp. 399-406, 2016.

[24]. G. Mehta, R. P. Singh, and V. K. Yada, "Optimization of Combined Economic Emission Dispatch Problem using Artificial Bee Colony Method," International Journal on Cybernetics \& Informatics (IJCI), vol. 6, no. 1/2, pp. 107-118, 2017. 
Apinan Aurasopon, et al.

[25]. W. KHAMSEN and Ch. TAKEANG, "Hybrid of Lamda and Bee Colony Optimization for Solving Economic Dispatch," PRZEGLAD ELEKTROTECHNICZNY, vol. 9, pp.220-223, 2016.

[26]. E. H. Chowdhury and Saifur Rahrnan, "A Review of Recent Advances IN Economic Dispatch,” IEEE Transactions on Power Systems, vol. 5, no. 4, pp. 1248-1259, 1990.

[27]. W. Sa-ngiamvibool, S. Pothiya and I. Ngamroo, "Multiple tabu search algorithm for economic dispatch problem considering valve-point effects," Electrical Power and Energy Systems, vol. 33, pp. 846-854, 2011.

[28]. R. Rahmani, M. F. Othman, R. Yusof, and M. Khalid, "Solving Economic Dispatch Problem using Particle Swarm Optimization by an Evolutionary Technique for Initializing Particles," Journal of Theoretical and Applied Information Technology, vol. 46, no. 2, pp. 526-536, 2012.

[29]. M. Basu and A. Chowdhury, "Cuckoo search algorithm for economic dispatch," Energy, ELSEVIER, vol. 60, pp. 99-108, 2013.

[30]. Babak Jeddi and Vahid Vahidinasab, "A modified harmony search method for environmental/economic load dispatch of real-world power systems," Energy Conversion and Management, ELSEVIER, vol.78, pp. 661-675, 2013.

[31]. Wael T. Elsayed and Ehab F. El-Saadany, "A Fully Decentralized Approach for Solving the Economic Dispatch Problem," IEEE Transactions on Power Systems, vol. 30, no. 4, pp. 2179-2189, 2015.

[32]. S. Sayah, A. Hamouda, "A hybrid differential evolution algorithm based on particle swarm optimization for non-convex economic dispatch problems," Applied Soft Computing journal home, vol. 13, Issue 4, 1608-1619, 2013.

[33]. B. Mandal, P.K. Roy, S. Mandal, "Economic load dispatch using krill herd algorithm," International Journal of Electrical Power \& Energy Systems, vol. 57, pp. 1-10, 2014.

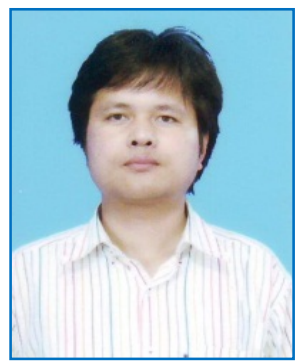

Apinan Aurasopon (Non-member) He received his B.Eng. degree in Electronic Engineering form the Northeastern College, Khon Kaen, Thailand, in 1995, his M.Eng. and Ph.D. degree in Electrical Engineering from the King Mongkut's University of Technology Thonburi, Bangkok, Thailand, in 2003 and 2007, respectively. In the past, he was a lecturer at the Department of Electrical Engineering, Faculty of Engineering, Burapha University, Chonburi, Thailand in 2007. In 2008 he has been a lecturer at the Department of Electrical Engineering, Faculty of Engineering, Mahasarakham University, Mahasarakham, Thailand.

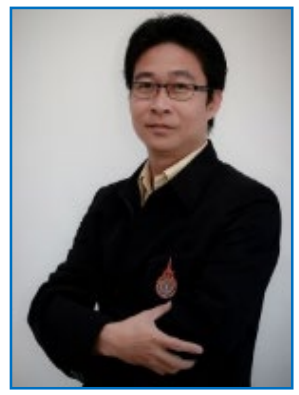

Chiraphon Takeang (Non-member) He received a B.S. degree in Technical Education in Electrical Engineering from the King Mongkut's Institute of Technology North Bangkok, Thailand, in 2003, him M.S. degree in Electrical Engineering from the Rajamangala University of Technology Lanna, Chiang Mai, Thailand, in 2015 and he holds a Ph.D. in Electrical and Computer Engineering, Mahasarakham University, where he has interests in electronics agriculture, smart farms, electric power systems and economic load dispatch. 